\title{
Using a coupled inductor controlled by fuzzy logic to improve the efficiency of a Buck converter in a PV system
}

\author{
Nabil Abouchabana ${ }^{1, *}$, Mourad Haddadi $^{2}$, Abdelhamid Rabhi ${ }^{3}$, and Ahmed El Hajjaji ${ }^{3}$ \\ ${ }^{1}$ LSMF laboratory, University of Laghouat, BP 37G, Ghardaia Road, Laghouat 03000, Algeria \\ ${ }^{2}$ LDCCP Laboratory, E N P, 10 avenue H. Badi BP 182 El Harrach Algiers, Algeria \\ ${ }^{3}$ MIS Laboratory. University of Picardie Jul. Verne, 33 rue Saint Leu.80039 Amiens Cedex1, France
}

\begin{abstract}
Photovoltaic generators $(\mathrm{PVG})$ produce a variable power according to the solar radiation $(\mathrm{G})$ and temperature $(\mathrm{T})$. This variation affects the sizing of the components of DC / DC converters, powered by such PVG, and make it difficult. The effects may differ from one component to another. The main and critical one is presented by the inductor, the element that stores the energy during sampled periods. We propose in this work an auto-adaptation of these inductor values to maintain optimal performance of the power yield of these converters. Our idea is to replace the inductor by a coupled inductor where this adjustment is made by the addition of an adjustable electric field in the magnetic core. Low current intensities come from the PVG supply the second inductor of the coupled inductor through a circuit controlled by a fuzzy controller (FC). The whole system is modeled and simulated under MATLAB/SIMULINK for the control part of the system and under PSPICE for the power part of the system. The obtained results show good performances of the proposed converter over the standard one.
\end{abstract}

\section{Introduction}

The purpose of the present paper is to find a topology designed to photovoltaic applications to ensure minimum losses whatever climate changes. A Photovoltaic generators (PVG) are characterized by non-linear I (V) characteristics with different points of maximum power (MPP) $[1,2]$. These characteristics depend on the level of radiation $(G)$ and the temperature of PV cells that compose it $[1,2,3]$. To address the latter influence, specific control laws were designed and developed since 1968 to now [3, 4], to allow these PVG to produce their maximum electrical power. This type of control is called Maximum Power Point Tracking (MPPT) $[4,5]$.

The main function performed by these controls is to ensure, at all times, a perfect adaptation between the PVG and load by making the operating point in the maximum power point (MPP), this adaptation is assured by a DC/DC converters (figure 1) [5].

\footnotetext{
*Corresponding author: n.abouchabana@lagh-univ.dz
} 
The problem now is that the power at the input of the converter changes depending to diverse parameters such as solar radiation and temperature [1-5], which causes a deterioration of the converter power yield because this yield is maximum only for the presizing of the DC/DC converter [6].

The aim of the paper is to propose a method allows us to maintain the converter power yield as maximum as possible when any change comes in the power input of the DC/DC converters used in PV applications.

The paper organizes as follows: as a starting point, we focus on a standard PV system with standard DC/DC converter commanded by an MPPT controller as presented in figure (1) to calculate the power yield of the DC/DC converter used in this application for various radiation levels (figure 3). We can improve its operating in his PPM using simulation tests. We have used the 'Simulink' environment of MATLAB to simulate different parts (solar panel, P\&O-MPPT), the DC-DC converter is simulated using the PSpice, SLPS bloc is used (see figure 2). After a discussion of the power yield result in section 2, Section 3 presents our proposed topology to improve the power yield. Simulation results for DC/DC converter using a coupled inductor on PV application and conclusions are provided in sections 4 and 5 respectively.

\section{PV System with standard Buck converter}

Photovoltaic systems have become globally accepted as a practical and feasible tool to generated power. One of the major problems of PV systems is that the output power of PV panels is highly dependent on solar radiation and ambient temperature [1-3]. Hence, loads cannot be directly connected to the output of PV panels. To force the PV generator to work as possible in its optimum regime, the commonly adopted solution is to introduce a static converter [5], which acts like source-load adapter as shown in figure 1.

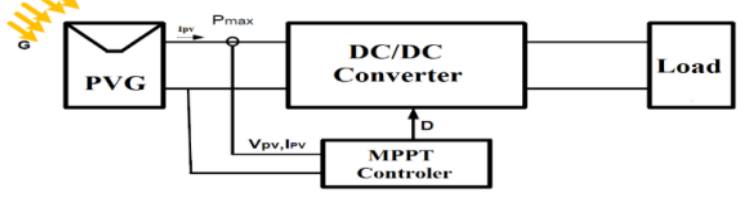

Fig.1. Elementary chain of a PV conversion used a DC/DC converter controlled by MPPT controller.

The MPPT controller delivers the action of appropriate control to track the maximum power point at any time. The choice of the conversion structure is performed depending on the load [2]. The P\&O method is an MPPT algorithm used in the field of MPPT technics. $\mathrm{P} \& \mathrm{O}$ method requires measures on the PVG output voltage Vpv and its output current Ipv. It detects the MPP and gives us the output voltage where the power is maximum $[2,3,5]$.

In our application, we use a buck topology for the DC/DC converter, which is controlled by a P\&O-MPPT controller, as an adaptation between the PVG and the load; the Buck electrical circuit is presented in figure 2.

The pre-sizing of the components in this Buck depends on: input current $i_{i n}$, and input voltage $V_{i n}$. Components to size are: inductor $\mathrm{L}$, capacitors $\mathrm{C}_{1}$ and $\mathrm{C}_{2}$, selection of the diode $\mathrm{D}$, and the switching transistor $\mathrm{K}$. When components $\mathrm{C}, \mathrm{D}$ and $\mathrm{K}$ are well chosen the energy loss in them will be neglected [6]. The element will influence on the Buck losses is the inductor $L$ because its sizing depends directly on the input Voltage and current $\left(V_{\text {in }}, i_{i n}\right)$. The Buck converter proposed in this paper is designed to operate in the continuous conduction modes (CCM) which means that the inductor current is always higher than zero. The inductance value is presented by equation (1) [6]. 


$$
L_{\min }=\alpha^{2}(1-\alpha) \frac{V i}{0,1 . f . I i}
$$

Where $L_{\min }$ is the minimum inductance, $\alpha$ is the duty cycle, $V_{i}$ is the input voltage, $I_{i}$ is the input current, and $f$ is the frequency of the PWM signal.

\subsection{DC/DC converter power Yield}

The Power yield « $\eta$ » of a system is defined by the ratio of output energy to the input energy. It is always between 0 and 1 . A system is more effective if its Power yield tends to 1 [7]. The Power yield of the DC/DC converter is defined and calculated by:

$$
\eta=\frac{P_{\text {Load }}}{P_{P V}}
$$

\subsection{Simulation tests}

In simulations tests, we have used the Orcad environment of PSpice to simulate the buck converter as figure 2 shows. We use ORCAD because it is based on the SPICE industry standard and provides access to model libraries developed by manufacturers. Also by using PSpice we can use the PSpice Model Editor to model a magnetic core from a datasheet.

The whole system is simulated by using the co-simulation between Orcad and Simulink, SLPS bloc is introduced. SIMULINK is used to implement control part of the system, see figure 2 .

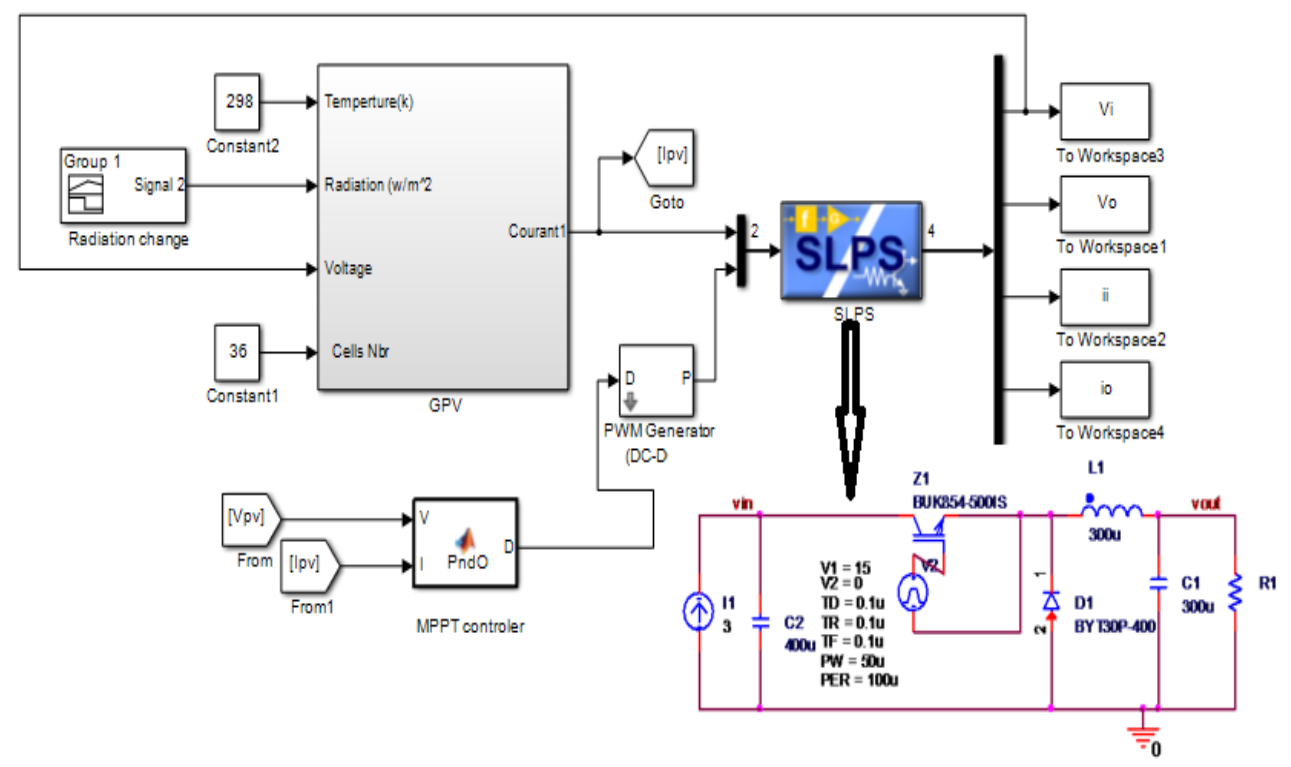

Fig. 2. Used Simulation Blocs.

The Simulation Blocs components are: PV panels as a power source (made by mathematical model $[8,9])$, DC-DC buck converter implemented using PSPICE that is considered as an adaptation bloc between PVG and the load, in figure 2 implemented circuit in PSpice for the SLPS Bloc is presented. The system is controlled using a P\&O algorithm which is modeled in Simulink . 


\subsection{Improvement of the MPPT controller and Power yield calculation}

Figure 3 presents the curve of radiation change used in simulation and efficiency of the $\mathrm{P} \& \mathrm{O}$ controller used in our application for different solar radiation changes. After assembling the whole system, we calculate the Power yield of the DC/DC converter used for different level of radiation, the trace of power yield is presented also in figure 3 .
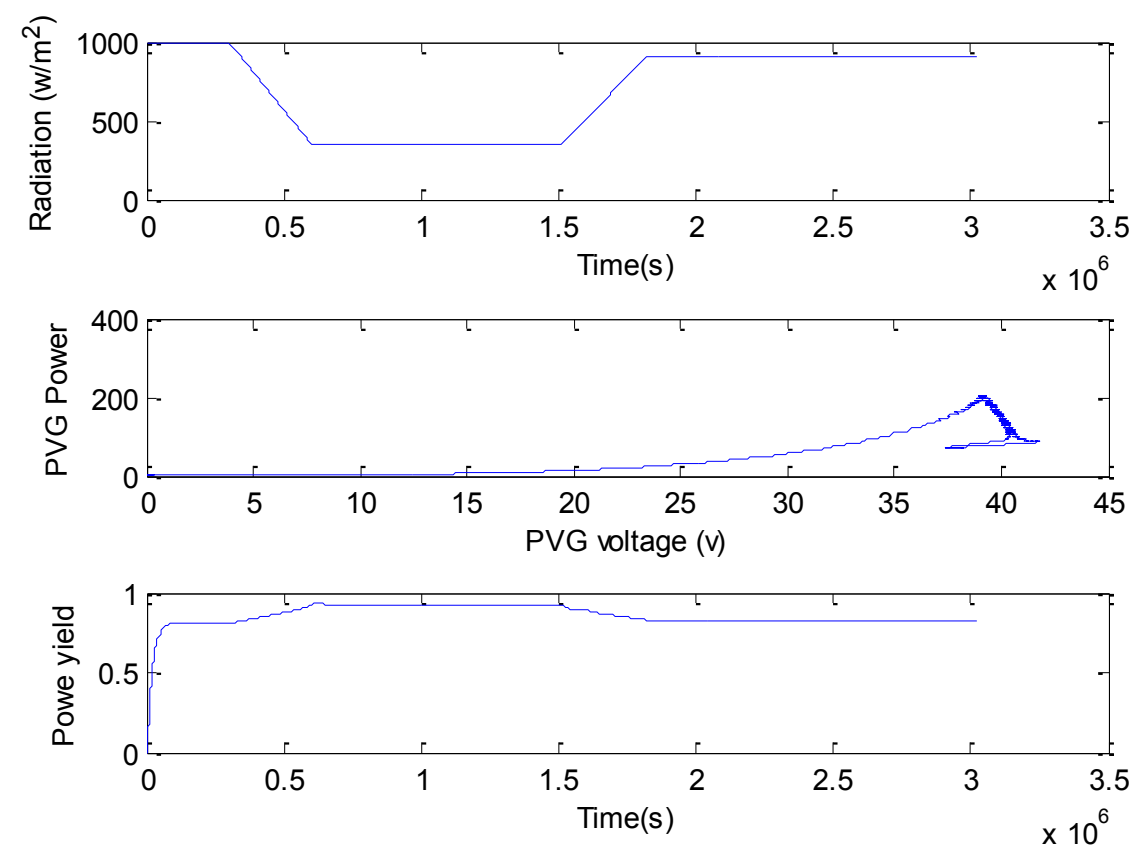

Fig. 3. Radiations curve, $\mathrm{P}=\mathrm{f}(\mathrm{V})$ characteristics, Power yield.

We remark that there is similarity, in shape, between the power curve and the radiation curve. The system tracks the maximum power the GPV can produce under the measured radiation. It is known that the converter's power yield is maximum only around the nominal current that is used to size the convert's elements. When the current varies as in figure 3 (the current is the image of the radiation) the converter's power yield decreases and can't be maximum all the time and therefore the topology of the converter have to be improved. According to the above, we have to find a topology where the power yield of a converter is almost maximum and constant regardless to the input power.

\section{Proposed DC/DC converter}

The sizing of conventional dc-dc converter elements is heavily based on the nominal input value. In table 1, a pre-sizing of inductor values for different current values is given using equation (1).

Table 1. Sized inductor value for different current values.

\begin{tabular}{|c|c|c|c|c|c|c|c|c|c|}
\hline $\mathrm{I}(\mathrm{A})$ & 0.5 & 1 & 1.5 & 2 & 2.5 & 3 & 3.5 & 4 & 4.5 \\
\hline $\mathrm{L}(\mu \mathrm{H})$ & 1100 & 530 & 353 & 265 & 212 & 177 & 167 & 133 & 118 \\
\hline
\end{tabular}


In case of PV applications where the current varies steadily, the sizing of the inductor becomes difficult and using only one value of the inductor results in more power loss. In the following section we propose a solution to fix the mentioned problem

\subsection{Proposed solution}

According to Table 1, we remark that the values of inductor in a DC/DC converter using in PV application must change depends on current input changes.

To adjust the inductor values automatically, in order to maximize the power yield of these converters, an external circuit has been added. The new topology is presented in figure 4. In the proposed topology, a coupled inductor is used instead of a simple inductor where (L1) value is adaptive to the input current $\left(\mathrm{I}_{\mathrm{PVG}}\right)$. The current $\mathrm{I}_{2}$ that flows through in (L2) is controlled to generate a variable electromagnetic field and therefore changing the L1 value. The reference current $\mathrm{I}_{2 \text { ref, }}$, that $\mathrm{I} 2$ must follow, is calculated basing on the measured

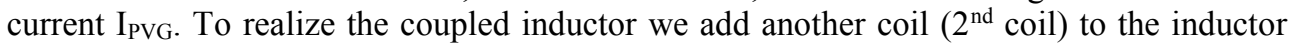
magnetic core used for inductor of the DC/DC converter ( $1^{\text {st }}$ coil). The two coils operate in mutuality between them and are connected as in figure 4 . The tow electromagnetic fields created by the tow coils constitute the total electromagnetic filed in the core.

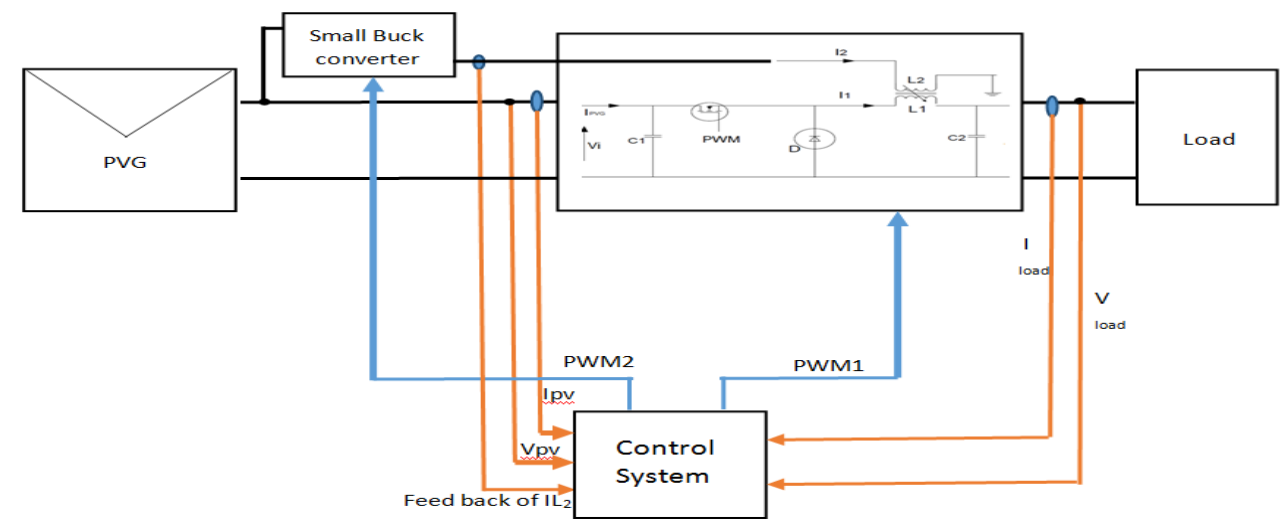

Fig. 4. PV System using Buck converter with a coupled inductor.

After sizing base on the reference [11], the coupled inductor in our application is composed of the magnetic core ETD 49 and a copper winding.

It is known that in $\mathrm{B}=\mathrm{f}(\mathrm{H})$ characteristic, the linear region can be long or short depending on the inductor core size [12]. When the core increases in size, the maximum current, which can be reached in the linear region, increases in value [12].

In the conventional power converters, the inductor operation point needs to meet the linear region of the B-H curve under all operating conditions $[6,12]$.

\subsubsection{Inductor and coupled inductor}

The following equations present the relationship between electromagnetic field and flux and inductance [10].

$$
\varphi=B . S
$$

Where B is the magnetic flux, and $\mathrm{S}$ is the cross section area $\left[\mathrm{m}^{2}\right]$. 


$$
B=H \cdot \mu
$$

Where $H$ is the electromagnetic field and $\mu$ is the permeability $[\mathrm{H} / \mathrm{m}]$

$$
\varphi_{T}=\frac{N^{2} . i}{R}
$$

Where: $R$ is the magnetic reluctance, $N$ is turns numbers and $\mathrm{I}$ is the flows current

Any change in the electromagnetic fields results in a change in the inductors values. Inductance $L$ is defined as the ratio between total flux $\varphi_{T}$ and current $i$ associated to a winding $N[10]$.

$$
L=\frac{\varphi_{T}}{i}
$$

For the coupled inductor: L1 and L2 are the self-inductance for the two coils and $M$ is the mutual inductance between L1 and L2. If the fluxes are additive $M$ is positive, and if the fluxes are subtractive $M$ is negative [10]. Sign (M) $=\mathrm{a}$

$$
\left\{\begin{array}{l}
\varphi_{1 T o t}=\varphi_{11 T o t}+a \cdot \varphi_{12 T o t}=L_{1} i_{1}+M i_{2} \\
\varphi_{2 T o t}=a \cdot \varphi_{21 T o t}+\varphi_{22 T o t}=M i_{1}+L_{2} i_{2}
\end{array}\right.
$$

Where $\varphi_{1 T o t}$ and $\varphi_{2 T o t}$ are the flux generated by L1 and L2 respectively.

The flux is proportional to the current that flows into the inductor $[12,13]$. In our proposed converter structure, the inductor core is sized to operate in the linear region where the maximum current, that flows into the inductor, do not exceed one third $(1 / 2)$ of the nominal PVG current. This means that the inductor will operate in the saturation zone when the nominal PV current flows into it, and therefore another solution is required.

Our proposed solution is to add another coil in order to generate a second flux in the magnetic core. Depending on the direction of the second flux, the later can be added or subtracted from the first one $[6,13]$. The total flux is the result of the addition or subtraction in the magnetic core using the second coil and to avoid saturation figure 5.

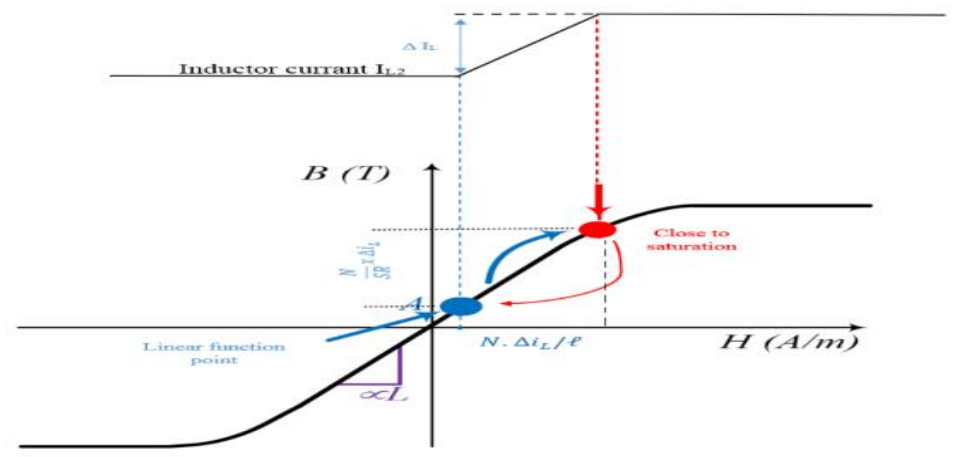

Fig. 5. Inductor current of a conventional Boost and Corresponding B-H curve.

Where $L$ is inductance, $l$ is core length, $N$ is number of turns in the winding and $\Delta i_{L}$ is the current change.

\subsubsection{SPICE modeling of ETD49 N67 core from datasheet}

The PSpice model of a core can be obtained from the B-H curve of ferrite from the datasheet and from the geometrical data of the core. We use Model Editor of PSpice to 
model the core used for the coupled inductor in simulation of the circuit presented by figure 4. A calculation data of its parameters will be done, and a Model of Core ETD49 will be created and able to use.

\section{Simulations tests}

To validate our idea simulations tests have been carried out. Two systems are simulated. One uses a standard BUCK as presented in figure 2. The second uses a coupled inductor as figure 4 shows. We used two similar PV panels model. Two model PSpice circuit for the SLPS bloc: one for the standard Buck circuit and the other for the Buck with the coupled inductor. The two inductors are made by the same magnetic core (ETD49), and the same resistive load is used.

A fuzzy controller (FC) controls the small buck converter presented in figure 4. FC is used to control the inductor currant $i_{2}$, this currant must have the same value as currant reference comes from theory calculation basing on PV currant measurement $i_{P V G}$. We have chosen the buck topology because the output current of a buck is always greater than its input current, so we can ensure that our second windings consume low current intensities even when $i_{2}$ has intensities greater than the powered current of buck input.

\subsection{Design of the Fuzzy controller}

The average output current of the PVG changes according to irradiation. When the latter is increased, the operating point of the inductor in the B-H curve changes and getting close to saturation zone and vice versa. The value of the inductor in this case must follow currant changes. From this observation, the idea of fuzzy control came up. Considering the error between the current $\left(i_{2}\right)$ and its reference $\left(i_{r e f}\right)$ comes from the calculation basing on $i_{1}$ sensing value, we can decide whether incrementing, decrementing or keeping unchanged the value of the duty cycle. One of the advantages of this strategy is that only one measurement is needed; which is of the inductor currant $i_{2}$. The fuzzy algorithm has been chosen with two linguistic input variables (error (E) and error change (DE)). Each variable has five membership functions. The output of the algorithm represents the value that we should add to the duty cycle. The fuzzy rules are summarized in Table 2.

Table 2. Fuzzy controller rules.

\begin{tabular}{|c|c|c|c|c|c|}
\hline DE $\backslash$ & NB & NS & $Z$ & PS & PB \\
\hline NB & PB & PS & PS & NS & NB \\
\hline NS & PB & PS & $Z$ & NS & NB \\
\hline Z & PB & PS & Z & NS & NB \\
\hline PS & PB & PS & Z & NS & NB \\
\hline PB & PB & PS & PS & NS & NB \\
\hline
\end{tabular}

NB: Negative Big, NS: Negative Small, PB: Positive Big, PS: Positive Small, Z: Zero

The figure 6 shows the result of the FC: reference current $\left(I_{r e f}\right)$ in red color and measurement current $\left(I_{\text {meas }}\right)$ in blue. We remark that The FC shows a good tracking performance. 


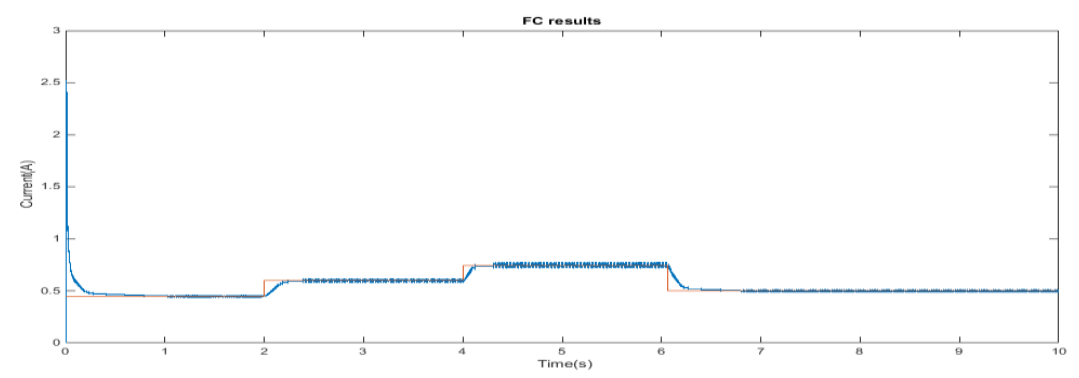

Fig. 6. Current through $2^{\text {nd }}$ coil and its reference.

\subsection{Simulation results}

To verify the advantages of the introduced concept, the powers yield of the system with and without coupled inductor are compared in figure 7 under same operating conditions.

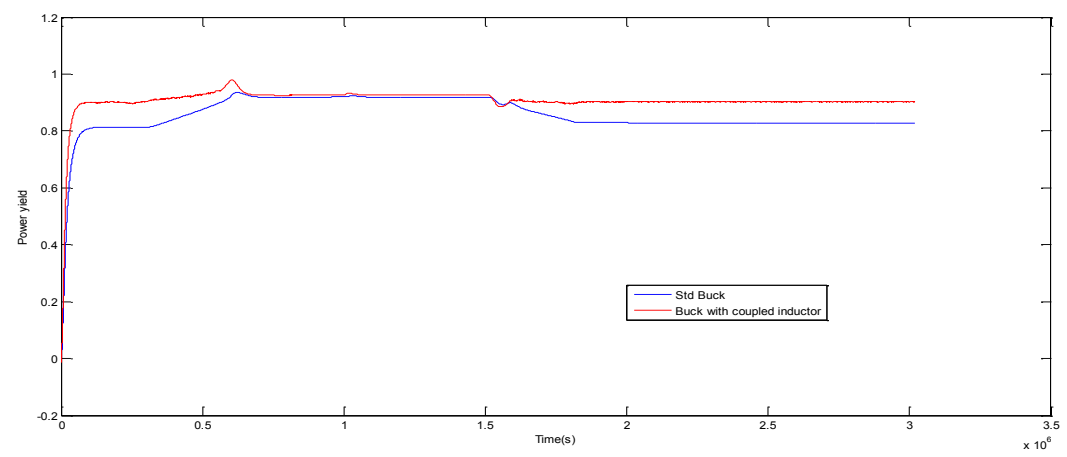

Fig. 7. Comparing power yield.

The results show that the power yield with coupled inductor is improved. We note that the curves of the power yield with coupled inductor (red curve) and without coupled inductor (blue curve) figure 7 meet at a certain stage. It is the stage where current in inductor have the value of pre-sizing.

\section{Conclusions}

The Buck converter with coupled inductor was analyzed and evaluated for PV application in this paper. Coupled inductor provides some advantages. Firstly, the lower inductor characteristic can be obtained (core size) by adding a second winding. Secondly, coupled inductor improves power yield of the DC/DC converter in PV application. A fuzzy controller is used to control the current in the second coil of the coupled inductor. The main advantage of the fuzzy controller is that no prior knowledge is needed about the system parameters and that only one measurement is required. The simulation of the proposed structure allowed us to improve Power yield of the DC/DC converter used in PV system.

\section{References}

1. J.A.R. Hernanz, J.J.C. Martn, I.Z. Belver, J.L. Lesaka, E.Z. Guerrero, E.P. Prez, (ICREPQ'10), Granada, 1-5, ( 2010) 
2. M. Ramli, S. Twaha, K. Ishaque, Y. Al-Turki, RSE Reviews 67, 144 (2017)

3. D. Verma, S. Nema, A.M. Shandilya, S. K. Dash, RSE R 54, 1018-1034 (2016)

4. A.F. Boehinger, IEEE AES-4, 1, 102-111 (1968)

5. J. Santos, F. Antunes, A. Chehab, C. Cruz, S. E. 80, 772-778 (2006)

6. M.H. Rashid, Power Electronics Handbook, (California, Academic press, 2001)

7. G. Chateigner, M. Boës, D. Bouix, J. Vaillant, D. Verkindère, Manuel De Génie Electrique, DUNOD, ISBN 978-2-10-048499-7

8. C.S. Chiu, IEEE Transactions on EC 25, 4, 1123-1132 (2010)

9. M. Park, I.K. Yu, Proc. IECON, 2040-2045 (2004)

10. Techniques de l'ingénieur, «Circuits magnétiques - Principes» (10August 2010)

11. http://schmidt-walter-schaltnetzteile.de/smps_e/abw_smps_e.html

12. P.L. Wong, Q. Wu, P. Xu, B. Yang, F.C. Lee, IEEE, 0-7803-5864-3 (2000)

13. M. Hirakawa, Y. Watanabe, M. Nagano, K. Andoh, S. Nakatomi, S. Hashino, T. Shimizu, IEEE, 978-1-4244-2893-9 (2009) 